\title{
Circuit
}

Musiques contemporaines

\section{Introduction. Écosystèmes sonores}

\section{Emanuelle Majeau-Bettez et Maxime McKinley}

Volume 29, numéro 3, 2019

Bozzini, Molinari, Quasar : trio de quatuors

URI : https://id.erudit.org/iderudit/1066482ar

DOI : https://doi.org/10.7202/1066482ar

Aller au sommaire du numéro

Éditeur(s)

Circuit, musiques contemporaines

ISSN

1183-1693 (imprimé)

1488-9692 (numérique)

Découvrir la revue

Citer ce document

Majeau-Bettez, E. \& McKinley, M. (2019). Introduction. Écosystèmes sonores.

Circuit, 29(3), 5-8. https://doi.org/10.7202/1066482ar d'utilisation que vous pouvez consulter en ligne.

https://apropos.erudit.org/fr/usagers/politique-dutilisation/ 


\title{
Introduction. Écosystèmes sonores
}

\author{
Emanuelle Majeau-Bettez \& Maxime McKinley
}

L'écosystème de la scène montréalaise des musiques contemporaines vit, au moins depuis les années $1950^{1}$, une évolution constante et de plus en plus foisonnante. Les trois organismes de ce milieu qui disposent des plus importants moyens sont, sans doute, la Société de musique contemporaine du Québec (SMCQ, fondée en 1966), l'Ensemble contemporain de Montréal (ECM+, fondé en 1987) et le Nouvel Ensemble Moderne (NEM, fondé en 1989). Ce sont là trois piliers aussi durables qu'incontournables. Assez rapidement toutefois, d'autres organismes ont fleuri et, parmi eux, quelques groupes de musique de chambre. Aujourd'hui encore, Montréal voit régulièrement apparaître (et parfois disparaître) des formations de chambre de divers acabits, mais qui ont en commun d'être vouées à la création. Dans la collection de Circuit, la SMCQ, l'ECM+ et le NEM occupent un espace conséquent. Au cours des dernières années, par exemple, leurs $50^{\mathrm{e}}, 30^{\mathrm{e}}$ et $25^{\mathrm{e}}$ anniversaires (respectivement) ont été soulignés ${ }^{2}$. Les ensembles chambristes évoqués dans le présent numéro sont tout aussi dignes d'attention, car ils jouent un rôle certes différent, mais (et en partie pour cette raison même) absolument essentiel dans l'écosystème. Leur contribution génère, notamment, un rayonnement international accru de la créativité musicale québécoise qu'ils défendent et, inversement, de nombreuses découvertes locales de ce qui se fait ailleurs dans le monde à l'heure actuelle. Par ailleurs, sans eux, les compositeurs québécois disposeraient de considérablement moins d'options pour leurs projets. Ces groupes chambristes n'ont évidemment pas l'exclusivité de ce genre de rôle, toutefois le fait d'être des organisations plus «petites» comporte les avantages de ses inconvénients, ce qui singularise leur apport. En effet, à bien des égards, elles sont aussi plus agiles, plus flexibles, souvent moins lourdes sur le plan logistique et moins coûteuses financièrement, ce qui n’est pas sans présenter de sérieux avantages dans certaines situations, par

1. On pense au désormais célèbre concert de Serge Garant, François Morel et Gilles Tremblay à la salle Saint-Sulpice du Conservatoire de Montréal, le $1^{\text {er }}$ mai 1954. 
3. Au sens, positif, de l'agilité (qui n'est pas à confondre avec celui de superficialité). Voir, à ce sujet, Italo Calvino (1989), Leçons américaines: aide-mémoire pour le prochain millénaire, "Légèreté", p. 17-58, Paris, Gallimard.
4. Rappelons, au sujet des fonctionnements "démocratiques" en musique, les réflexions du violoniste Clemens Merkel (du Quatuor Bozzini) concernant les modes décisionnels en contexte de quatuor, exposées dans son article "Le quatuor à cordes: une géométrie sociale", publié en 2013 dans Circuit (vol. 23, n 3, p. 29-37). Emanuelle Majeau-Bettez a repris et développé ces réflexions dans son article "Cool Control, Occam, and Océan : The Radigue and Bozzini Game", paru en 2017 dans Les Cahiers de la société québécoise de recherches en musique (vol. 18, nº 1, p. 51-66).
5. Qui avait, d'ailleurs, invité Quasar à se joindre à elle pour l'occasion.
6. Denys Bouliane avait dirigé pour l'occasion, en collaboration avec Jean Lesage, une brochure intitulée Présence de la musique québécoise: 22 portraits instantanés (Montréal, s.é., 1999). La même année, Jean Boivin était revenu, dans les pages de Circuit, sur cet événement majeur dans un texte intitulé «Le festival Présences 99: une fenêtre ouverte sur la nouvelle réalité québécoise" (vol. 10, nº 2, p. 39-50).

exemple lorsque vient le temps d'expérimenter étroitement et longuement avec des compositeurs, de faire des tournées, d'investir des lieux qui diffèrent de la salle de concert traditionnelle, ou encore d'enregistrer des disques. Ces particularités forment une zone extrêmement riche à explorer, à laquelle nous avons décidé de consacrer un dossier thématique de Circuit. Par ailleurs, ce sont des aspects particulièrement pertinents en 2019, à une époque où plusieurs modes opératoires, en bien des sphères, tendent vers davantage de «légèreté ${ }^{3}$ » et de décentralisation. Ainsi, en arts, tout paraît tourner moins exclusivement qu'autrefois autour de quelques capitales culturelles mondiales (Paris, New York ou plus récemment Berlin, par exemple). Le rapport au «pouvoir» semble aussi, dans plusieurs cas, tendre à la décentralisation. Même dans le monde des orchestres symphoniques, parfois jugé conservateur et rigide, la tendance est aux chefs à l'écoute des musiciens, sans excès d'autoritarisme, travaillant dans un esprit proche... de la musique de chambre ${ }^{4}$.

Il est intéressant ici de faire un exercice de mémoire concernant l'effort titanesque fourni par Denys Bouliane en 1999, dans le cadre du festival Présences de Radio France, pour la reconnaissance internationale de la musique québécoise. Ce sont pas moins de vingt compositeurs (et deux ensembles, en l'occurrence le NEM et la SMCQ ${ }^{5}$ ) qu'il a voulu, en quelque sorte et en simplifiant un peu, faire entrer par la grande porte. L'impact ne fut peut-être pas celui espéré, l’accueil fut parfois mitigé, mais ce geste énergique force l'admiration ${ }^{6}$. S'il nous est permis d'emprunter au golf une métaphore, cette démarche aurait bien pu ressembler, sur le plan de la reconnaissance internationale, à un spectaculaire «trou d'un coup». Les vases communicants entre scènes locales et internationales tels que façonnés par les divers groupes de chambre montréalais s'apparentent davantage, pour leur part, à un long et patient travail de «fourmis» (chantant comme des cigales, pour compliquer d'un paradoxe la fable de La Fontaine). Et ce travail de fond, constant, démontre de plus en plus que, comme dit l'adage populaire, "petit train va loin ». La lecture de ce dossier thématique permet, entre autres choses, de bien s'en rendre compte.

Dans le cadre d'un numéro de revue, il fallait bien sûr (et non sans regrets) délimiter nos choix afin de trouver un équilibre convenable entre, d'une part, un éventail un tant soit peu varié et, d'autre part, suffisamment d'espace pour aller un peu plus loin qu'un simple coup d'œil superficiel. Les critères qui ont servi de crible pour établir ce périmètre sont les suivants: 1) il devait s'agir de quatuors montréalais 2) encore actifs aujourd'hui, 3) cela depuis vingt ans ou davantage, et 4) se consacrant principalement aux musiques de création contemporaines. C'est ainsi que nous en sommes venus à ce « trio de 
quatuors » : les quatuors à cordes Bozzini (fondé en 1999) et Molinari (fondé en 1997), ainsi que le quatuor de saxophones Quasar (fondé en 1994)7. Plutôt que de tenter des imbrications et des logiques d'enchaînement plus ou moins artificielles, nous leur consacrons ici chacun, par ordre alphabétique, un diptyque. Ainsi, Emanuelle Majeau-Bettez signe un article «en deux temps » sur le Quatuor Bozzini. Ce vaste travail d'enquête aux allures de fresque nous mène d'abord à la rencontre des membres du Quatuor Bozzini, puis à celle de quelques-uns de leurs collaborateurs privilégiés par le truchement de propos recueillis (dans l'ordre: Éliane Radigue, Phill Niblock, Marc Boivin, Nicole Lizée, Simon Martin et James O'Callaghan). Le second diptyque est consacré au Quatuor Molinari. D’abord, sa fondatrice et directrice artistique, Olga Ranzenhofer, nous livre un texte personnel, autobiographique, qui traverse l'histoire de cet ensemble, des débuts jusqu'à aujourd'hui. Ce vivant témoignage est suivi d'une étude rigoureuse d'Alexis Raynault sur trois pans de la discographie de ce quatuor, ceux consacrés à R. Murray Schafer ${ }^{8}$, Jean Papineau-Couture et Petros Shoujounian. Enfin, le dernier diptyque est consacré au quatuor de saxophones Quasar'. Jimmie LeBlanc y propose un riche parcours de l'évolution de ce quatuor depuis 25 ans, en particulier quant à leurs modes de collaborations avec les compositeurs. Le deuxième volet consiste en un Cahier d'analyse signé par Anthony Tan, qui offre une étude de cas détaillée de l'une de ces collaborations et de son résultat, en se penchant sur l'œuvre not thinking about the elephants (2018) de la compositrice canadienne - maintenant établie en Allemagne - Annesley Black.

Il est arrivé que ces trois quatuors joignent leurs forces. Par exemple, le Quatuor Bozzini et Quasar ont fait équipe pour la création de Tags (2018) de Joane Hétu ${ }^{10}$. Sans jouer directement ensemble, les quatuors Bozzini et Molinari se sont parfois succédé sur scène lors d'un même événement, par exemple lors d'un «marathon» de l'édition 2017 du festival Montréal/ Nouvelles musiques, dans lequel les premiers jouaient Jean Lesage et les seconds, John Zorn ${ }^{11}$. On pourrait également penser à la collaboration de Quasar avec l'étiquette de disques du Quatuor Bozzini: Collection $\mathrm{QB}^{12}$. Comme nous l'avons vu, ces trois quatuors participent à un même écosystème et partagent un contexte général commun. Mais chacun est aussi, voire d'abord, un écosystème en soi et une voix singulière. C'est ce que nous avons souhaité mettre en valeur en leur consacrant chacun un diptyque. Au fond, un peu comme en contemplant longuement un objet impossible, on peut percevoir alternativement, dans ce numéro, aussi bien un seul que trois différents dossiers thématiques... Ou peut-être même quatre? Ce sont ces ambiguïtés qui nous ont incités à proposer à Camila $\mathrm{Paz}$ - artiste d'origine chilienne
7. Il importe de préciser à quel point il y a là une abondance et qu'il aurait été passionnant de parler également, par exemple, des participations à cet écosystème du Trio Fibonacci, de Sixtrum, de Transmission, ou d'ensembles plus jeunes tels que Paramirabo ou le duo Wapiti, et bien d'autres encore.

8. Rappelons ici le numéro de Circuit consacré au "quatuor à cordes selon Schafer", dirigé par Jean Portugais dans la foulée d'événements du Quatuor Molinari, en 1999, autour d'une prestation des sept premiers quatuors de Schafer (vol. 11, $\mathrm{n}^{\circ} 2$, 2000).

9. Des trois quatuors discutés dans ce numéro, Quasar est peut-être celui qui a occupé le moins d'espace dans les pages de Circuit jusqu'à maintenant. L'idée de ce numéro est d'ailleurs venue à l'esprit du rédacteur en chef de la revue dans la foulée d'une conversation (spontanée et informelle) avec Marie-Chantal Leclair, directrice artistique et générale de Quasar. Soulignons tout de même un texte de Cléo Palacio-Quintin sur deux disques de Quasar - Du souffle et De souffles et de machines, les deux sous étiquette Collection QB -, paru dans les Actualités du vol. 26, $n^{\circ} 2$ en 2016 ("Nouveautés en bref", p. 88-90).

10. Sur ce concert, voir: https:// www.levivier.ca/fr/calendrier/40778 (consulté le 7 août 2019).

11. " $3 \times 4=12$ : le marathon de quatuors". À noter qu'ils y étaient précédés du Quatuor Capitano. Voir: http://smcq.qc.ca/smcq/fr/ evenement/37269/3_4_12_Le_ marathon_de_quatuors (consulté le 7 août 2019).

12. Voir: https://www.collectionqb com/fr/accueil (consulté le 7 août 2019). 
13. Ce numéro est aussi illustré par quelques photos d'archives de ce trio de quatuors.

14. Après Claude Vivier, Gilles Tremblay, Ana Sokolović, Denis Gougeon, John Rea et José Evangelista. Voir: http://smcq.qc.ca/smcq/ $\mathrm{fr} /$ hommage/2019 (consulté le 7 août 2019). Tous ces hommages ont été soulignés par Circuit.

15. On retracera aisément d'autres opus de la revue dans lesquels cet enjeu est abordé: https://revuecircuit. ca/collection/ (consulté le 7 août 2019).

16. Ensemble dont Makdissi-Warren est la fondatrice et directrice artistique.

17. Notons que Makdissi-Warren n'est pas la seule musicienne que Joséphine Bacon inspire. Ce poème a également été mis en musique par Sébastien Sauvageau (voir le disque de maintenant établie à Montréal - de dessiner pour nous des objets impossibles, demande à laquelle elle a répondu avec enthousiasme et habiletée ${ }^{3}$.

Ainsi, en complément à ce dossier thématique (ou comme quatrième dossier, c'est selon), nous retrouvons les Actualités. Bien que cette rubrique soit généralement hors thème, nous la consacrons cette fois entièrement à Katia Makdissi-Warren, à qui la SMCQ dédie sa Série hommage 2019-2020 ${ }^{14}$. D'ailleurs, il ne serait pas saugrenu d'affirmer que le dossier thématique de ce numéro, à défaut de relever d'une Série hommage, constitue une manière d'hommages en série. Et signalons au passage qu'une création de MakdissiWarren pour Quasar est prévue dans le cadre de cette Série hommage. Le travail de cette compositrice concerne aussi de près les nombreuses redéfinitions de l'échiquier mondial en ce début de xxI ${ }^{\mathrm{e}}$ siècle, comme le démontrent la chronologie de son parcours et le diagramme des ramifications de ses influences culturelles préparés par Noémie Pascal. Ce «portrait» poursuit ainsi une discussion récurrente, dans la collection de Circuit, sur les rencontres interculturelles en création musicale contemporaine ${ }^{15}$. Pour sa part, Vicky Tremblay nous offre un panorama de l'univers de Makdissi-Warren, ponctué d'entretiens avec elle. Badih El Haji nous mène à la rencontre d'un mentor très important pour la musicienne dans son apprentissage de la musique arabe, le Père Louis Hage. Gordon Williamson nous partage ses impressions et réflexions autour du disque Saimaniq de l'ensemble Oktoécho ${ }^{16}$, réalisé en collaboration avec des chanteuses inuites. Nous passons ensuite à un texte de Katia Makdissi-Warren elle-même, soit un extrait de sa thèse de doctorat déposée en 2007, portant sur l'enjeu (vibrant d'actualité) de la «cohérence d'une œuvre métissée ». Ce dossier se termine avec les mots émouvants de la poète innue Joséphine Bacon, qui a été une collaboratrice (très appréciée) de la compositrice. Nous reproduisons son poème «Tu es musique » qui évoque, en français et en innu-aimun, des «nuages sans frontières ${ }^{17}$ ».

Comme toujours, rien n’est exhaustif ni épuisé dans ces pages. Les numéros de Circuit sont bien souvent des amorces de conversations - ni plus mais ni moins! En ce sens, nous vous rappelons que nous sommes toujours heureux de vous lire. N'hésitez pas à communiquer avec nous, que ce soit par courriel, sur notre page Facebook ou sur Twitter ${ }^{18}$.

En terminant, nous tenons à remercier chaleureusement les quatuors Bozzini, Molinari et Quasar, ainsi que Katia Makdissi-Warren et la SMCQ, pour leurs précieuses collaborations à ce numéro.

\footnotetext{
Bonne lecture!

Montréal, août 2019
} 